Article

\title{
Analysis of Complete Genome Sequence of Acinetobacter baumannii Strain ATCC 19606 Reveals Novel Mobile Genetic Elements and Novel Prophage
}

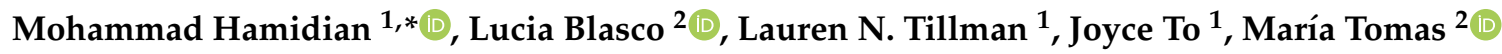 \\ and Garry S. A. Myers ${ }^{1}$ \\ 1 The iThree Institute, University of Technology Sydney, Ultimo 2007, NSW, Australia; \\ Lauren.Tillman@student.uts.edu.au (L.N.T.); joyce.to@uts.edu.au (J.T.); garry.myers@uts.edu.au (G.S.A.M.) \\ 2 Microbiology Department-Research Institute Biomedical A Coruña (INIBIC), Hospital A Coruña (CHUAC), \\ University of A Coruña (UDC), 15495 A Coruña, Spain; lucia.blasco@gmail.com (L.B.); \\ MA.del.Mar.Tomas.Carmona@sergas.es (M.T.) \\ * Correspondence: mohammad.hamidian@uts.edu.au
}

Received: 2 November 2020; Accepted: 21 November 2020; Published: 24 November 2020

\begin{abstract}
Acinetobacter baumannii isolate ATCC 19606 was recovered in the US prior to 1948. It has been used as a reference and model organism in many studies involving antibiotic resistance and pathogenesis of A. baumannii, while, until recently, a complete genome of this strain was not available. Here, we present an analysis of the complete 3.91-Mbp genome sequence, generated via a combination of short-read sequencing (Illumina) and long-read sequencing (MinION), and show it contains two small cryptic plasmids and a novel complete prophage of size $41.2 \mathrm{~kb}$. We also characterised several regions of the ATCC 19606 genome, leading to the identification of a novel cadmium/mercury transposon, which was named Tn6551. ATCC 19606 is an antibiotic-sensitive strain, but a comparative analysis of all publicly available ST52 strains predicts a resistance to modern antibiotics by the accumulation of antibiotic-resistance genes via plasmids in recent isolates that belong to this sequence type.
\end{abstract}

Keywords: Acinetobacter baumannii ATCC 19606; complete genome; plasmid; antibiotic resistance; ST52 and Multi-Locus Sequence Typing (MLST)

\section{Introduction}

Acinetobacter baumannii is a Gram-negative opportunistic pathogen that has emerged in recent decades as a global challenge to healthcare. It causes pneumonia, wound, soft tissue and bloodstream infections and is a major cause of infections in intensive care units (ICUs) [1]. Eradicating A. baumannii is problematic mainly because of its natural resistance to extreme environmental conditions and its ability to acquire a range of antibiotic resistance genes [2-4]. A. baumannii strains have highly plastic genomes, which elevates a need for knowledge of the genomic features at the strain level.

A. baumannii ATCC 19606 was recovered in a urine sample prior to 1948 in the US and is one of the earliest isolates available in current collections [5]. It is one of the most antibiotic susceptible strains available to researchers and, hence, has been widely used in studies both as a reference and model strain for studying the emergence and evolution of resistance, pathogenesis and for the discovery of new antibacterial targets $[6,7]$.

Several draft genomes are available for ATCC 19606 (GenBank acc. nos. JMRY01000000 [8], ACQB01000000 and APRG01000000). We deposited the first complete genome for ATCC 19606 in GenBank in October 2019 (GenBank acc. no. CP045110), but in quick succession, two further complete 
genomes for ATCC 19606 were also made publicly available (GenBank acc. nos. CP046654 and AP022836). One of these genomes was completed using PacBio only (GenBank acc. nos. CP046654) [9], and the latest genome was completed using a combination of Illumina MiniSeq (short-read) and MinION (long-read) sequence data (GenBank acc. nos. AP022836) [10]. Zhu et al. performed a comparative analysis of ATCC 19606 with 98 diverse A. baumannii genomes belonging to a variety of sequence types (ST) [9], while the study of Tsubouchi et al. included no analysis of this genome [10].

Here, we report a complete genome for ATCC 19606 generated from a combination of Illumina MiSeq and Oxford Nanopore (MinION) sequence data, as well as an analysis of its genomic features and other ST52 strains. To complement our genome and strengthen the confidence in inferences made from our genomic analysis, we also performed a set of phenotypic assays for antibiotic resistance, mercury resistance, biofilm production and phage lytic ability. Our complete genome for ATCC 19606 includes two cryptic plasmids and a prophage never previously described. Here, we aimed to provide a detailed characterisation of the genetic features and evolutionary relationships of ATCC 19606 and other ST52 strains to further understand the genomic features and mechanisms for the development of antibiotic resistance.

\section{Materials and Methods}

\subsection{Antibiotic Resistance Profile and Resistance to Mercury}

Antibiotic resistance profile of A. baumannii ATCC 19606, which was obtained from the ATCC culture collection and kindly supplied by Prof. Ruth Hall of the University of Sydney, Australia, against 22 antibiotics was determined using the standard disc diffusion method, as previously described [11]. Antibiotic discs tested were: ampicillin $(25 \mu \mathrm{g})$, ampicillin/sulbactam $(10 / 10 \mu \mathrm{g})$, cefotaxime $(30 \mu \mathrm{g})$, ceftazidime $(30 \mu \mathrm{g})$, imipenem $(10 \mu \mathrm{g})$, meropenem $(10 \mu \mathrm{g})$, piperacillin/tazobactam $(100-10 \mu \mathrm{g})$, timentin (ticarcillin/clavulanic acid) $(75 / 10 \mu \mathrm{g})$, streptomycin $(25 \mu \mathrm{g})$, spectinomycin $(25 \mu \mathrm{g})$, sulfamethoxazole $(100 \mu \mathrm{g})$, trimethoprim $(5 \mu \mathrm{g})$, kanamycin $(30 \mu \mathrm{g})$, neomycin $(30 \mu \mathrm{g})$, gentamicin $(10 \mu \mathrm{g})$, amikacin $(30 \mu \mathrm{g})$, tobramycin $(10 \mu \mathrm{g})$, netilmicin $(30 \mu \mathrm{g})$, rifampicin $(30 \mu \mathrm{g})$, tetracycline $(30 \mu \mathrm{g})$, ciprofloxacin $(5 \mu \mathrm{g})$ and nalidixic acid $(30 \mu \mathrm{g})$. Resistance and susceptibility were interpreted according to the Clinical and Laboratory Standards Institute (CLSI) guidelines for Acinetobacter spp. [12] and calibrated dichotomous sensitivity disc diffusion assay (CDS) (http://cdstest.net/) when a CLSI breakpoint for Acinetobacter spp. was not available (e.g., for netilmicin, streptomycin, spectinomycin, sulfamethoxazole, nalidixic acid and rifampin). Antibiotic resistance profile was determined using two individual colonies of ATCC 19606. All antibiotics were purchased from OXOID, UK.

To examine whether ATCC 19606 is resistant to mercury, 10 fresh colonies were patched onto L-agar supplemented with $20-\mu \mathrm{g} / \mathrm{mL} \mathrm{HgCl} 2$ followed by overnight incubation at $37^{\circ} \mathrm{C}$ and visual inspection for the presence and absence of growth, as previously described [13].

\subsection{Static Biofilm Assay and Quantification of Biofilm Production Using Fluorescence Microscopy}

Biofilm formation was measured using the standard crystal violet assay in 96-well plates, as described previously [14]. Briefly, ATCC 19606 and controls were grown overnight at $37{ }^{\circ} \mathrm{C}$ in Cation-adjusted Muller-Hinton broth (CaMHB; Sigma-Aldrich, St. Louis, MO, USA). All cultures were then normalised to OD600 of 0.05 ( 1 in 100 dilution) and then $100 \mu \mathrm{L}$ of cells transferred to each well of a 96-well plate. Growth and media-only controls were also included. The 96-well plate was covered with Aeroseal (Thermo Fisher ${ }^{\mathrm{TM}}$, Waltham, MA, USA) and incubated for $24 \mathrm{~h}$ and a second plate for $48 \mathrm{~h}$ at $37^{\circ} \mathrm{C}$. After the incubation, plates were washed with PBS buffer (Phosphate-buffered Saline) using an automatic plate washer (BIO-TEK ELx405TM, Winooski, VT, USA), followed by staining with $150 \mu \mathrm{L}$ of $0.2 \% \mathrm{CV}$ (Crystal Violet) per well and incubating at room temperature for $1 \mathrm{~h}$ on an automatic rocker. The washing step was repeated, followed by adding $170 \mu \mathrm{L}$ of $33 \%$ acetic acid to each well and incubating at room temperature on a rocker for $30 \mathrm{~min}$. Absorbance at $600 \mathrm{~nm}$ was measured using a plate reader (Tecan M200, Männedorf, Switzerland). 
Fluorescence microscopy was used to visualise biofilm microcolonies and to measure their properties. Briefly, $1 \mathrm{~mL}$ of normalised cell culture (as prepared for CV assay) was grown for 24 or $48 \mathrm{~h}$ at $37^{\circ} \mathrm{C}$ in CaMHB in WPI ${ }^{\mathrm{TM}}$ dishes. After the incubation, dishes were washed gently with saline and stained with $1 \mathrm{~mL}$ of 2-uM Syto-9 for $1 \mathrm{~h}$, then washed again and fixed with $1 \mathrm{~mL}$ of freshly prepared $4 \%$ PFA for $1 \mathrm{~h}$ prior to imaging. DeltaVision Elite microscope (Applied Precision) was used to acquire Z-stack wide-field fluorescence images; 5 randomly selected fields of view (FOV) were captured in the FITC channel at 20× magnification. All images were analysed using IMARIS softwarev.9.5.1 with the Biofilm Analysis tool (https://imaris.oxinst.com).

All biofilm assays were performed with technical triplicates and included three additional strains, AB0057 (member of global clone 1), ACICU (member of global clone 2) and ATCC 17978 (widely used reference strain). Results were analysed and plotted using the Prism GraphPad software v8.2.0. 2.3. whole-genome sequencing and assembly.

\subsection{Genome Sequencing}

Whole-cell genomic DNA of ATCC 19606 was isolated using the DNeasy ${ }^{\mathrm{TM}}$ UltraClean Microbial Kit (Qiagen ${ }^{\mathrm{TM}}$, Germantown, MD, USA) from cells grown overnight at $37^{\circ} \mathrm{C}$ in LB inoculated from a single colony. Library preparation and barcoding for Illumina MiSeq and MinION (Oxford Nanopore Technologies ${ }^{\circledR}$, Oxford, UK) sequencing was performed by the UTS Core Sequencing Facility at the ithree Institute, as described previously $[15,16]$. Illumina sequencing generated 1,024,087 paired-end short reads with 50-fold coverage and an average length of $250 \mathrm{bp}$; MinION generated a total of 10,687 reads with an N50 of $18.2 \mathrm{kbp}$ and 30-fold coverage. FastQC (v.0.11.9) (https://bioinformatics. babraham.ac.uk/projects/fastqc/) and Filtlong (v.0.2.0) (https://github.com/rrwick/Filtlong) were used to check the quality of Illumina and MinION reads, respectively. Filtlong filtered long reads by quality and length. The high=quality Illumina and MinION reads were assembled de novo using a hybrid assembly approach with the Unicycler program (v0.4.7) [17]. Protein coding, rRNA and tRNA gene sequences were annotated using Prokka [18], and the resistance and polysaccharide loci (outlined below) were annotated manually.

\subsection{Phylogenetics and Sequence Analysis}

Phylogenetic relationships of all available ST52 strains were examined by generating a core genome alignment. Briefly, Illumina sequence reads for all isolates were mapped to ATCC 19606, which was also used as a reference using snippy (available at https:/github.com/tseemann/snippy) to generate a whole-genome alignment. Snippy mapped all reads to the reference genome using bwa v0.7.12 and minimap 2 v 2.0 using default parameters. High-quality variant sites were called using SAMtools v1.3.1.24 with standard-quality filtering, as described previously (10). Single-nucleotide differences (SNDs) in recombinant regions were identified and removed using Gubbins v2.1.025 (33) with default parameters, including the default taxa filtering percentage of $25 \%$. A maximum likelihood phylogenetic tree was inferred from the resulting recombination-filtered alignment using RAxML (v.8) with the GAMMA model. The tree was visualised and annotated using the R package ggtree v1.12.027. Recombination blocks were plotted against the phylogenetic tree in R v.3.5.2. using the ggtree v. 1.16.6 and ggplot2 v.3.2.1 packages and PlotTree available at https://github.com/katholt/plotTree. Bootstrap values were calculated using ten independent runs of RAxML with 1000 bootstraps, which each gave near-identical results.

A range of bioinformatic tools were used for the sequence analysis. A local database of the genome sequence of the strains studied here was created, and sequence analysis was done locally using the standalone BLAST program available at ftp://ftp.ncbi.nlm.nih.gov/blast/executables/blast+/LATEST/. The Artemis Comparison Tool (ACT) 16.0.0 [19] was used to visualise comparisons of large regions performed by Standalone BLAST. SnapGene Viewer v 4.2.4 was used to visualise, manipulate and export the sequence data. 
Protein coding and gene features studied here were annotated manually using a combination of BLASTP (http://blast.ncbi.nlm.nih.gov/Blast.cgi), Pfam (http://pfam.xfam.org/) and UniProt (https: //www.uniprot.org) searches, as described elsewhere.

The IS-Finder (https://www-is.biotoul.fr/) database was used to identify and analyse insertion sequences (IS).

The PHASTER (http://phaster.ca) database was used to look for integrated phage genomes. The phage genome sequences were further analysed and annotated using the RAST server (Rapid Annotation using Subsystem Technology; https://rast.nmpdr.org), BLAST (https://blast.ncbi. nlm.nih.gov/Blast.cgi) and the HHPRED bioinformatics toolkit (https://toolkit.tuebingen.mpg.de/tools/ hhpred). Figures were drawn to scale using SnapGene Viewer ${ }^{\circledR} \mathrm{v}$ 4.2.4 and reconstructed using the Inkscape v.1.0 program.

\subsection{Data Availability}

The complete genome sequence was deposited in DDBJ/ENA/GenBank under the accession no. CP045110 (chromosome), CP045108 (p1ATCC 19606) and CP045109 (p2ATCC 19606). Illumina and MinION sequence reads were deposited in the sequence read archive database under the accession numbers SRR10248709 and SRR10248708, respectively.

\subsection{Phage Induction and Isolation}

In order to induce the expression of the prophage and its release from the bacteria, an overnight culture of A. baumannii strain ATCC 19606 was diluted 1:100 in LB broth and incubated at $37^{\circ} \mathrm{C}$ and $180 \mathrm{rpm}$. When the culture reached $0.5 \mathrm{OD}(600 \mathrm{~nm})$, mitomycin $(10 \mu \mathrm{g} / \mathrm{mL})$ was added to the culture and incubated until the culture was cleared by lysis. After $30 \mathrm{~min}$ of incubation at room temperature in the presence of chloroform (1\%), the culture was centrifuged at $3000 \times g$ for $15 \mathrm{~min}$, and the supernatant with the isolated phage was recovered and filtered through a 0.45-um filter.

\subsection{Phage Concentration and Preparation for Transmission Electron Microscopy (TEM)}

To concentrate the phage previously isolated, the lysate was incubated with $\mathrm{NaCl}$ to a final concentration of $0.5 \mathrm{M}$ and left on ice for $1 \mathrm{~h}$. The suspension was centrifuged at $3400 \times g$ for $40 \mathrm{~min}$ at $4{ }^{\circ} \mathrm{C}$, and the supernatants were transferred to sterile tubes. PEG $6000(10 \% w / v)$ (polyethylene glycol) was added, dissolved and incubated overnight at $4{ }^{\circ} \mathrm{C}$. Bacteriophages were then precipitated at 3400 $g$ for $40 \mathrm{~min}$ at $4{ }^{\circ} \mathrm{C}$ and resuspended in SM buffer (0.1-M NaCl, 1-mM MgSO 4 and 0.2-M Tris-HCl, $\mathrm{pH} 7.5)$.

For the visualisation of the phage by TEM, the samples of the phage in SM buffer were negatively stained with $1 \%$ aqueous uranyl acetate in grids and then examined on a JEOL JEM-1011 electron microscope.

\subsection{Phage Host Range}

The host range of the phage was established by applying a previously established spot test [20] to a set of diverse 19 clinical strains of $A$. baumannii, of different STs, isolated from Spanish hospitals during the GEIH-REIPI Spanish Multicentre A. baumannii Study II 2000-2010, GenBank Umbrella project PRJNA422585. Briefly, an overnight culture of the A. baumannii strain host was diluted 1:100 and incubated at $37{ }^{\circ} \mathrm{C}$ and $180 \mathrm{rpm}$ until it reached an optical density of 0.5 (OD600nm). TA soft medium (agar $0.4 \%$ ) supplemented with $10-\mathrm{mM} \mathrm{CaCl}_{2}$ was previously prepared and maintained at $50^{\circ} \mathrm{C}$. Four millilitres of the TA soft medium was mixed with $200 \mu \mathrm{l}$ of the A. baumannii culture, and the mix was poured on the top of a TA plate (agar 1.5\%). When the soft medium was solidified, a spot of $10 \mu \mathrm{l}$ of the phage suspension was dropped on the plate, and when adsorbed, it was incubated at $37^{\circ} \mathrm{C}$. After $24 \mathrm{~h}$, the plates were checked to detect the presence of a halo, which indicated lysis. 


\section{Result}

\subsection{Antibiotic Resistance Profile}

The resistance profile for ATCC 19606 to individual antibiotics has been tested in previous studies. However, to draw a complete picture of ATCC 19606's resistance profile and to phenotypically confirm the genome reported here, ATCC 19606 was tested against 22 antibiotics representing all clinically important antibiotic classes. Overall, ATCC 19606 is susceptible to a wide range of antibiotics, including nalidixic acid, ciprofloxacin and many antibiotics in the $B$-lactam and aminoglycoside families (Table 1). It was found to be resistant to ampicillin, streptomycin spectinomycin, chloramphenicol and sulfamethoxazole (Table 1).

Table 1. Antibiotic resistance profile of ATCC 19606.

\begin{tabular}{ccc}
\hline Antibiotic Family & Antibiotic & $\begin{array}{c}\text { Growth Inhibition Zone } \\
\text { (Diameter in } \mathbf{~ m m} \mathbf{~}^{\mathbf{1}}\end{array}$ \\
\hline \multirow{3}{*}{ B-lactams } & Ampicillin & $\mathbf{0}$ \\
& Cefotaxime & 15 \\
& Ceftazidime & 18 \\
& Ceftriaxone & 17 \\
& Imipenem & 19 \\
& Meropenem & 21 \\
& Ampicillin/Sulbactam & 19 \\
\hline \multirow{2}{*}{ Aminoglycosides } & Gentamicin & 14 \\
& Kanamycin & 15 \\
& Neomycin & 14 \\
& Tobramycin & 13 \\
& Netilmicin & 16 \\
& Amikacin & 15 \\
& Streptomycin & $\mathbf{7}$ \\
& Spectinomycin & $\mathbf{0}$ \\
\hline \multirow{2}{*}{ Fluoroquinolones } & Nalidixic acid & 13 \\
& Ciprofloxacin & 16 \\
\hline Tetracyclines & Tetracycline & 15 \\
\hline Chloramphenicol & Chloramphenicol & $\mathbf{0}$ \\
\hline \multirow{2}{*}{ Rynthesis Inhibitors } & Rifampicin & 14 \\
\hline
\end{tabular}

${ }^{1}$ Bold indicate antibiotics to which the resistance was observed.

\subsection{Complete Genome Sequence of ATCC 19606, Antibiotic Resistance Gene and Its Genomic Context}

To determine the complete genome sequence of ATCC 19606, we used a combination of Illumina and MinION sequence data. We used the long reads to generate the genome scaffold and $>50$ rounds of polishing using the Illumina reads, given the accuracy of Illumina reads, to minimise the sequencing errors in the final assembly. The genome sequence of ATCC 19606 was completed and submitted to GenBank in October 2019 (https:/www.ncbi.nlm.nih.gov/nuccore/CP045110). The final assembly consists of 3,981,968 bases, including the chromosome (3,981,941 bp; Figure 1) and two plasmids, p1ATCC19606 (7655 bp) and p2ATCC19606 (pMAC) (9540 bp), with a copy number of 11x and 13x for each plasmid, respectively. The chromosome of ATCC 19606 has an average GC content of 39.15\% (highest $51.66 \%$ and lowest $22.5 \%$ ) and encodes a total of 3693 putative proteins, 74 tRNAs and 6 rRNA regions. 


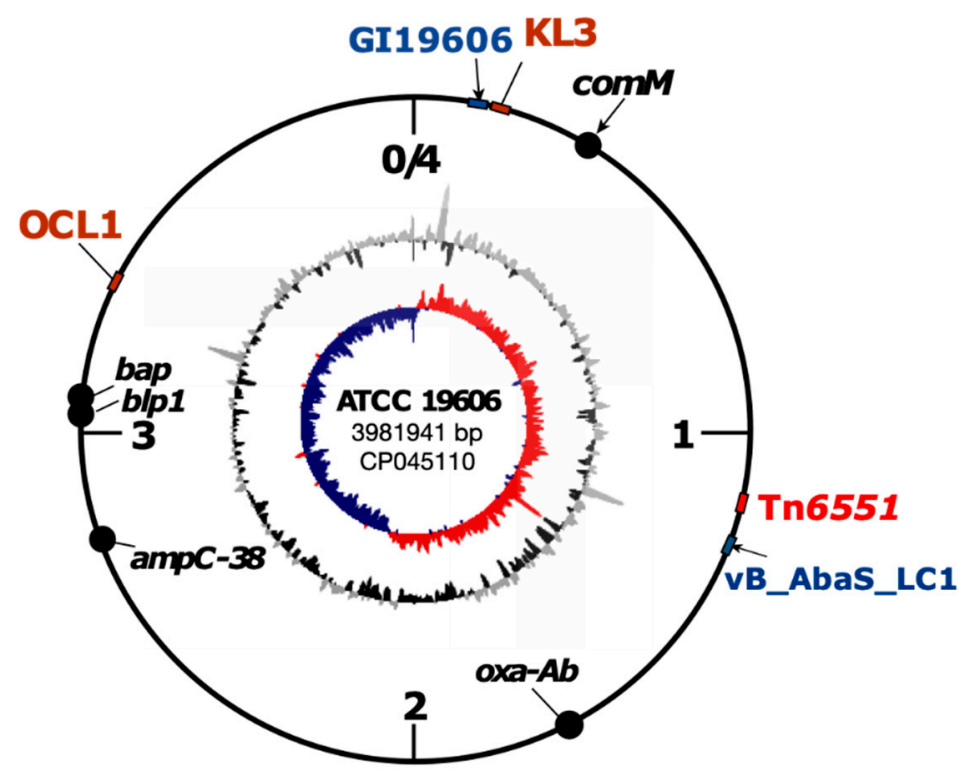

Figure 1. Schematic representation of the complete genome of Acinetobacter baumannii ATCC 19606. Important genes, genomic islands, transposons and surface polysaccharide loci (OC and K) are marked on the outermost ring representing the chromosome. The inner most ring represents the GC skew, and the middle ring represents the GC content of the ATCC 19606 chromosome.

Recently, the genome of ATCC 19606 was also completed by two other groups: (i) using the PacBio technology (Table 2) [9] and (ii) a combination of Illumina MiniSeq and MinION [10]. To examine the differences in the genomes generated by the three groups, we compared all three genomes. The chromosome sequence of our assembly is 1093-bp longer than that in CP046654 due to the absence of a copy of the insertion sequence ISAba11, which was found at positions 998797-999897 of our assembly. The two chromosome assemblies also differ by 50 single-nucleotide differences (SNDs) and small insertions/deletions of mainly one-five bases (50 bp in total) spread across the chromosome. The chromosome of ATCC 19606 deposited in GenBank acc. no. AP022836 differs from our assembly again by the absence of the additional ISAba11 found in our assembly, 270 SNDs and a substantial number of small insertions/deletions (1660 bp in total) of mainly up to 10 bases in the chromosome. Notably, there are far less differences between our assembly and that completed using PacBio by Zhu et al. [9] than the genome reported by Tsubouchi et al. [10]. The additional ISAba11 copy in our assembly is presumably due to IS movement, given that there is already another ISAba11 in all ATCC 19606 chromosomes, while SNDs and short insertions/deletions could be due to either sequencing/assembly errors, real mutations or, likely, a combination of both.

The complete genome of ATCC 19606 includes a single acquired antibiotic resistance gene, the sul 2 sulfonamide resistance gene, which confers resistance to sulfamethoxazole and accounts for the sulfamethoxazole resistance phenotype observed in this strain [21]. We previously predicted, using the publicly available draft genome sequences of ATCC 19606, a chromosomal genomic island in ATCC 19606 containing the sul2 gene in the GIsul2 genomic island [21]. This GI will be referred to as GI19606 hereafter. The complete ATCC 19606 genome reported here confirms the structure of GI19606 as a 36,157-bp genomic island located at bases 80477-116633 of the ATCC 19606 chromosome (GenBank acc. no. CP045110) spanning locus_ids FQU82_00080 to FQU82_00125 (Figure 1). The other two ATCC 19606 complete genomes also contain GI19606 [9,10]. 
Table 2. Properties of the complete ATCC 19606 genomes.

\begin{tabular}{|c|c|c|c|c|c|c|c|}
\hline $\begin{array}{c}\text { ATCC } 19606 \\
\text { Genomes }\end{array}$ & Length & GC\% & $\begin{array}{c}\text { Reading } \\
\text { Frames }\end{array}$ & $\begin{array}{l}\text { Sequencing } \\
\text { Technology }\end{array}$ & $\begin{array}{c}\text { Assembly } \\
\text { Program }\end{array}$ & GenBank no. & Ref. \\
\hline Chromosome & $3,981,941$ & 39.1 & 3727 & $\begin{array}{l}\text { Illumina } \\
\text { MiSeq \& } \\
\text { MinION }\end{array}$ & Unicycler & СР045110 & This study \\
\hline $\begin{array}{c}\text { p1ATCC } \\
19606\end{array}$ & 7655 & 33.3 & 14 & & & СР045108 & \\
\hline $\begin{array}{l}\text { p2ATCC19606 } \\
\text { (pMAC) }\end{array}$ & 9540 & 34.6 & 14 & & & СР045109 & \\
\hline $\begin{array}{l}\text { Chromosome } \\
\text { pMAC }\end{array}$ & $\begin{array}{c}3,980,848 \\
9540\end{array}$ & $\begin{array}{l}39.1 \\
34.6\end{array}$ & $\begin{array}{c}3709 \\
14\end{array}$ & & $\begin{array}{c}\text { SMRT } \\
\text { plasmidSPAdes }\end{array}$ & $\begin{array}{l}\text { СР046654 } \\
\text { СР046655 }\end{array}$ & [9] \\
\hline Chromosome & $3,978,812$ & & & $\begin{array}{l}\text { Illumina } \\
\text { MiSeq \& } \\
\text { MinION }\end{array}$ & Unicycler & AP022836 & [10] \\
\hline $\begin{array}{l}\text { pATCC } \\
19606-1\end{array}$ & 9408 & 34.6 & 14 & & & AP022837 & \\
\hline $\begin{array}{l}\text { pATCC } \\
19606-2\end{array}$ & 7631 & 33.3 & 12 & & & AP022838 & \\
\hline
\end{tabular}

ATCC 19606 is susceptible to third-generation cephalosporins and carbapenems, and consistent with this, no ISAba1 copy was found upstream of the intrinsic ampC and $o x a-A b$ genes. However, in our complete genome assembly, the ATCC 19606 chromosome contains a copy of the intrinsic ant(3")-IIa aminoglycoside resistance gene (located at bases 225025-225813; locus id FQU82_00218), accounting for the streptomycin and spectinomycin resistance phenotypes observed (Table 1). This intrinsic ant(3")-IIa aminoglycoside resistance gene is also present in the other two complete genomes.

\subsection{Tn6551, a Novel Mercury/Cadmium Transposon Found in ATCC 19606}

Given that genes conferring resistance to antibiotics and resistance to heavy metals can occur together on the same genetic element, we searched the complete genome of ATCC 19606 for genetic elements associated with heavy metal resistance. A set of mer resistance genes predicted to confer resistance to mercury were found on an approx. $6.5-\mathrm{kbp}$ region that was flanked by a set of cadmium/zinc resistance genes and a partial tnpA gene, which were identical to those in Tn6018 [3]. Tn6018 is a 3372-bp cadmium/zinc transposon that is often found embedded in the AbaR-type resistance islands in members of global clone 1 [3]. Tn6018 is flanked by 24-bp inverted repeats (IR) and generates 8-bp target site duplication upon insertion [3]. Further analysis of this region showed that this mercury/cadmium/zinc region in ATCC 19606 includes properties of a class II transposon and is related to Tn6018. Hence, this transposon was named Tn6551 (Figure 2). Tn6551 is a 6582-bp novel transposon bounded by 24-bp IRs and flanked by the $5^{\prime}$-ATTTTTTT-3' 8-bp target site duplications (TSD). Tn6551 is located at bases 1237446-1244027 of the ATCC 19606 chromosome (GenBank acc. no. CP045110) between FQU82_01194 (encoding a hypothetical protein) and the fic gene (FQU82_01201, encoding a putative adenosine monophosphate-protein transferase). In Tn6551, the $5^{\prime}$-end of the tnpA gene is interrupted by the merACPR genes (Figure 2). However, the other end of this mer module appears to be deleted, as the $\mathrm{IR}_{t n p}$ of Tn1696 could not be detected. Analysis of the mer region indicated that it is a hybrid Tn501/1696/5053 mer module (GenBank acc. no. Z00027: Tn501, Y09025: Tn1696 and L40585: Tn5053) with an $\mathrm{IR}_{1696}$ located at the $3^{\prime}$-end of merR. 

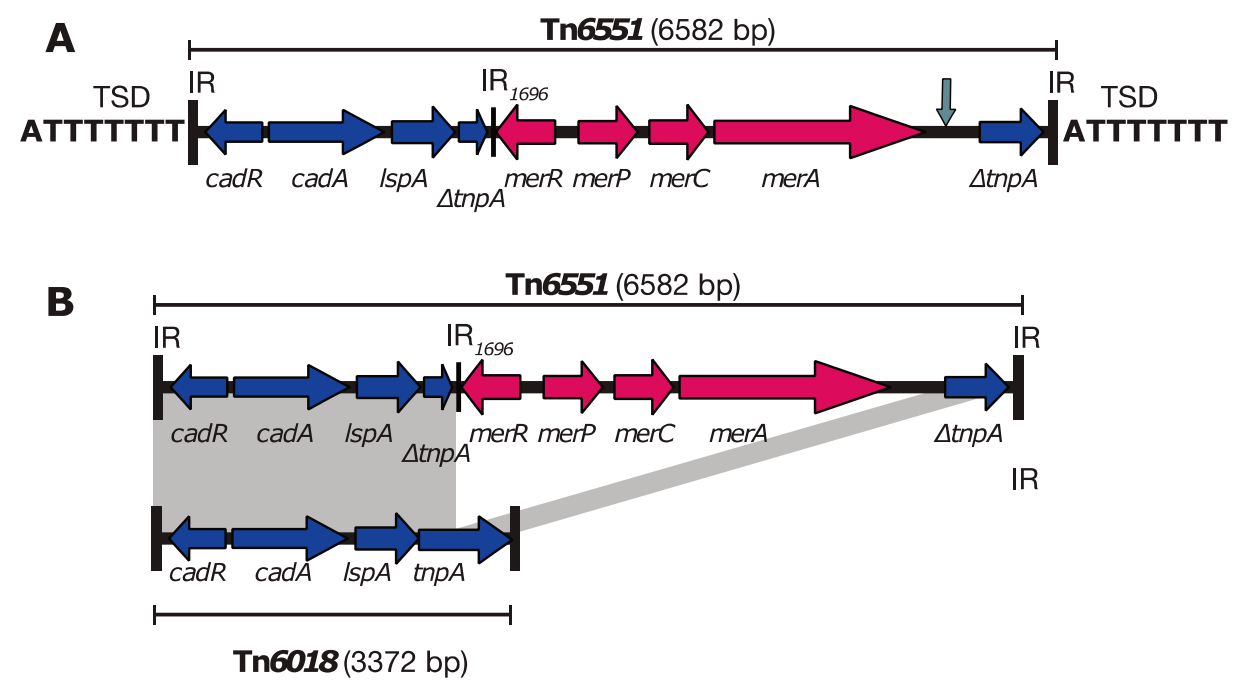

Figure 2. Genetic structure of $\operatorname{Tn} 6551$ (A) and its comparison to Tn6018 (B). Horizontal arrows indicate the extent and orientation of genes and open reading frames with their names shown below. Inverted repeats (IR) are indicated using black vertical lines. Target site duplications generated by Tn6551 are shown flanking this element in panel A and marked target site duplications (TSD). The vertical grey arrow indicates the position of the 96-bp deletion in Tn6551-v1.

ATCC 19606 was tested for mercury resistance; however, none of the colonies patched onto L-agar supplemented with $20-\mu \mathrm{g} / \mathrm{mL} \mathrm{HgCl} 2$ generated a visible colony, suggesting that this mercury operon is likely to be nonfunctional.

To track Tn6551, the GenBank nonredundant database was searched using the sequence of Tn6551 as a query. Tn6551 and/or variants of it were found in related strains, unrelated strains, different Acinetobacter species and a different bacterial genus. A copy of Tn6551 was found precisely in the same chromosomal location in an ST52 clinical strain recovered in 2015 in the US (strain ab736; Table 3). This suggests a link to ATCC 19606, given that both strains belong to the same sequence type (see a comparative analysis of ST52 strains below). An almost identical copy of Tn6551 (with 99.9\% DNA identity) was identified also in two other completely unrelated Acinetobacter strains, AB031 and BEC1-S18-ESBL-01 (Table 3). Notably, the latter belongs to a different species, A. pittii, suggesting the transfer of Tn6551 across species. In addition, a variant of Tn6551, which we named Tn6551-v1, was found in several strains belonging to different species of the Acinetobacter genus, indicating that both Tn6551 and Tn6551-v1 are widely spread (Table 3). Compared to Tn6551, the -v1 variant is 96-bp longer and was likely generated due to a separate deletion event truncating the hybrid mer501/1696/5053 module, leaving an additional $96 \mathrm{bp}$ of the mer module behind (vertical grey arrow in Figure 2A). Alternatively, it is possible that Tn6551 is a derivative of Tn6551-v1 that was generated as a result of a second deletion event; however, this could not be verified, as no intermediate structure is currently available in GenBank. Unexpectedly, a copy of Tn6551-v1 was also found in the chromosome of a Neisseria brasiliensis strain, N.177.16, recovered in 2016 in Brazil (Table 3). N.177.16 belongs to a completely different bacterial genus, suggesting its transposition across two different bacterial genera. 
Table 3. Distribution of Tn6551 and related transposons in Acinetobacter.

\begin{tabular}{|c|c|c|c|c|c|c|c|c|}
\hline Genus/Species & Strain & Date & Country & Source & ST (IP) & Tn & $\underset{5^{\prime}-3^{\prime}}{\text { TSD }}$ & $\begin{array}{c}\text { GenBank } \\
\text { no. }\end{array}$ \\
\hline A. baumannii & ATCC 19606 & $<1948$ & USA & Urine & 52 & 6551 & ATTTTTTT & СР045110 \\
\hline A. baumannii & ab736 & 2015 & USA & Blood & 52 & 6551 & ATTTTTTT & СР015121 \\
\hline A. baumannii & AB031 & 2010 & Canada & Blood & 638 & 6551 & АТTTTTTT & СР009256 \\
\hline A. pittii & BEC1-S18-ESBL-01 & 2018 & Japan & Water $^{2}$ & 457 & 6551 & ATTTTTTT & AP022302 \\
\hline A. baumannii & NCTC7364 & 2014 & UK & $\mathrm{nk}^{3}$ & 494 & $6551-v 1^{4}$ & ATTTTTTT & LT605059 \\
\hline A. indicus & Yy_1 & 2019 & China & Soil & - & 6551-v1 & ATTTTTTT & СР039031 \\
\hline A. junii & WCHAJ59 & 2015 & China & Sewage & - & 6551-v1 & ATTTTTTT & СР028800 \\
\hline A. nosocomialis & AC1530 & 2015 & Malaysia & Blood & - & $6551-v 1$ & ATAATTAA & А СР045560 \\
\hline $\begin{array}{l}\text { Neisseria } \\
\text { brasiliensis }\end{array}$ & N.177.16 & 2016 & Brazil & $\begin{array}{c}\text { Ulcer } \\
\text { exudate }\end{array}$ & - & $6551-v 1$ & ATGTATTT & $\mathrm{CP} 046027$ \\
\hline
\end{tabular}

We previously showed that DNA fragments containing an ISAba1-activated ampC gene or an entire genomic island could be acquired from an exogenous source via homologous recombination [22,23]. To examine whether homologous recombination is the mechanism for the Tn6551 and Tn6551-v1 exchange events, we analysed the chromosomal sequences flanking Tn6551 and the $-v 1$ variant. Analysis of the flanking sequences of Tn6551 showed that a $7044 \mathrm{bp}$ on the left and a $3934 \mathrm{bp}$ on the right of Tn6551 in ATCC 19606 were 100\% and 99.99\% (1-bp difference), respectively, identical to the corresponding sequences in AB301 (GenBank acc. no. CP009256). This suggests a possible homologous recombination exchange event of a 17,560-bp DNA fragment (including Tn6551) between ATCC 19606 and AB301 (Table 3). AB301 belongs to ST638, which is a very rare sequence type with only one complete genome available in GenBank. No other exchange event was detected, indicating that the acquisition of Tn6551 and Tn6551-v1 by different strains of Acinetobacter species occurred through multiple transposition events. In all but A. nosocomialis AC1530 and Neisseria brasiliensis N.177.16, Tn6551 and Tn6551-v1 are present in precisely the same chromosomal location, suggesting a preference for this particular chromosomal site. Nonetheless, the presence of Tn6551-v1 copies in completely different chromosomal locations in A. nosocomialis AC1530 and Neisseria brasiliensis N.177.16 indicates that these transposons could also target alternative genomic spots.

\subsection{ATCC 19606 Carries Two Cryptic Plasmids}

Our complete ATCC 19606 genome carries two cryptic plasmids, which are named p1ATCC19606 and p2ATCC19606 (pMAC). In addition to our assembly, the entire sequence of p1ATCC19606 is present in all available draft genomes of ATCC 19606 (GenBank acc. no. JMRY01000000, ACQB01000000 and APRG01000000) and in the complete genome of the Tsubouchi et al. group [10] (Table 2), but it is absent from the genome reported by Zhu et al. [9], likely due to sequencing/assembling error. p1ATCC19606 is a novel 7655-bp cryptic plasmid that encodes 14 open reading frames, one of which is a novel RepAci putative replication initiation protein (Figure 3). Its closest known match is RepAci2 (encoded by pABVA01; GenBank acc. no. FM210331) with 95\% aa identity. p1ATCC19606 also encodes the higAB toxin-antitoxin system, a prevalent toxin-antitoxin type in small plasmids of A. baumannii.

p1ATCC19606 (CP045108; 7655 bp)

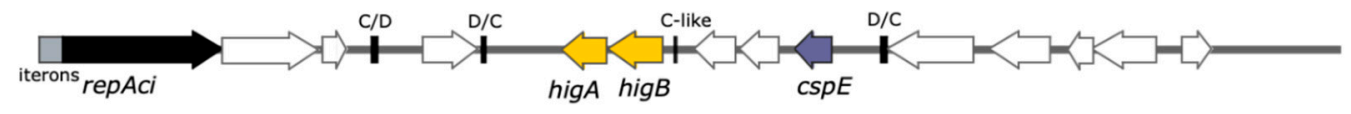

Figure 3. Genetic structure of p1ATCC 19606. Horizontal arrows indicate the extent and orientation of genes and open reading frames with their names shown below. White arrows indicate open readings with unknown functions. Small black vertical lines indicate pdif recombination sites, and the filled grey box upstream of the repAci gene indicates the iteron region. 
Recently, a number of $A$. baumannii plasmids have been shown to include pdif sites, consisting of inversely oriented binding sites for the XerC and XerD recombinases separated by 6 bp [13,24-26]. Here, our manual inspection of p1ATCC19606 identified three pdif sites (XerC/D at bases 1971-1998, XerD/C at bases 2613-2640 and XerD/C at bases 4973-5000), as well as an 11 bp that resembles a XerC-binding site at bases 3758-3768 of p1ATCC19606 (GenBank acc. no. CP045108; Figure 3). The pdif module, carrying hig $A B$ and flanked by $\mathrm{D} / \mathrm{C}$ and $\mathrm{D} / \mathrm{C}$ sites, is in a wide range of small plasmids (data not shown). The sequence of this plasmid in the genome completed by the Tsubouchi et al. group [10] is 24-bp shorter due to the absence of the C/D site of the module carrying a single orf. This might represent a sequencing/assembling error, as careful inspection of the flanking sequence did not reveal any evidence to explain the deletion. However, this could not be verified, as ATCC $19606^{\mathrm{T}}$ was not available.

p2ATCC19606, also known as pMAC, is a well-studied 9540-bp cryptic plasmid first reported in 2006 [27]. The sequence of p2ATCC19606 (pMAC) in our assembly and that sequenced by Zhu et al. [9] are identical to the original sequence of pMAC (GenBank acc. no. AY541809.1) (Table 2). However, the sequence of this plasmid is 132-bp shorter in the sequence reported by Tsubouchi et al. [10], again likely due to sequencing errors.

Our manual inspection of p2ATCC19606 (pMAC) identified five pdif sites, including XerC/D at bases 1868-1895, XerD/C at bases 2510-2537, XerD/C at bases 5226-5253, XerC/D at bases 6264-6291 and XerD/C at bases 6809-6836 of GenBank acc. no. CP045109 (picture not shown), which indicates that it consists of five pdif modules. This was not noted before.

\subsection{ATCC 19606 Carries a Novel Prophage, vB_AbaS_LC1}

Our analysis of the genomic sequence of ATCC 19606 established the presence of a 41,282-bp complete prophage. This prophage was located at the genomic position 1336408-1377688 bp (GenBank acc. no. CP045110; Figure 1) and had a GC content of 40.4\%. This prophage region encodes 59 open reading frames (Table 4 ), of which $41 \%$ have unknown functions; $10 \%$ have putative replication, recombination and synthesis functions; $17 \%$ are structural proteins; $5 \%$ involved in defence against the host; $6 \%$ involved in the lytic-lysogenic cycle and $5 \%$ have putative functions related to the entry and exit of the virus from the cell, including a lysozyme and a sialidase. These have potential as enzymes for phage therapy.

To find out if there were any homologues of the prophage that we identified, we first performed a homology analysis by BLAST of the prophage sequence. This showed the presence of an identical genomic region in the chromosome of A. baumannii strain Ab736 (GenBank acc. no. CP015121). Ab736 belongs to the sequence type ST52 and is, therefore, related to ATCC 19606. However, this genomic region is not annotated and/or reported as a prophage. Secondly, we performed a bacteriophage homology analysis by comparing the genomic sequence of the phage with the viral genomes deposited in the GenBank nonredundant database. No homology was obtained for any known phage, suggesting that the prophage we found in ATCC 19606 is a novel phage. We named this phage vB_AbaS_LC1. 
Table 4. Annotation and function of the 59 proteins identified in the phage vB_AbaS_LC1.

\begin{tabular}{|c|c|c|c|c|}
\hline Predicted Function & $\begin{array}{l}\text { Biological } \\
\text { Function }\end{array}$ & Protein Id ${ }^{1}$ & E-Value & Tool \\
\hline Hypothetical protein & Unknown function & QFQ04715.1 & $4.00 \times 10^{-177}$ & BLASTp \\
\hline Hypothetical protein & Unknown function & QFQ04716.1 & $5.00 \times 10^{-114}$ & BLASTp \\
\hline Hypothetical protein & Unknown function & QFQ04717.1 & $4.00 \times 10^{-53}$ & BLASTp \\
\hline Hypothetical protein & Unknown function & QFQ04718.1 & 0 & BLASTp \\
\hline Hypothetical protein & Unknown function & QFQ04719.1 & $1.00 \times 10^{-42}$ & BLASTp \\
\hline $\begin{array}{l}\text { RecA protein; Recombination, } \\
\text { Radio-resistance, DNA repair, } \\
\text { ATPase, DNA binding; HET: AGS; } \\
\text { 2.5A \{Deinococcus radiodurans\} } \\
\text { SCOP: d.48.1.1, c.37.1.11 }\end{array}$ & Recombination & QFQ04720.1 & $1.30 \times 10^{-10}$ & HHpred \\
\hline Hypothetical protein & Unknown function & QFQ04721.1 & $1.00 \times 10^{-73}$ & BLASTp \\
\hline $\begin{array}{c}50 S \text { ribosomal protein } \mathrm{L} 2,50 S \\
\text { Ribosome, bacterial ribosome, } \\
\text { proline-rich antimicrobial; }\end{array}$ & Protein synthesis & QFQ04722.1 & 0.3 & HHpred \\
\hline Hypothetical protein & Unknown function & QFQ04723.1 & $1.00 \times 10^{-102}$ & BLASTp \\
\hline $\begin{array}{c}\text { Helix-turn-helix } \\
\text { domain-containing protein }\end{array}$ & Lysogeny & QFQ04724.1 & 0 & BLASTp \\
\hline $\begin{array}{c}\mathrm{Cro} / \mathrm{Cl} \text { family transcriptional } \\
\text { regulator }\end{array}$ & Lysogeny & QFQ04725.1 & $7.00 \times 10^{-54}$ & BLASTp \\
\hline $\begin{array}{l}\text { XRE family transcriptional } \\
\text { regulator }\end{array}$ & Lysogeny & QFQ04726.1 & $4.00 \times 10^{-117}$ & BLASTp \\
\hline Hypothetical protein & Unknown function & QFQ04727.1 & $7.00 \times 10^{-64}$ & BLASTp \\
\hline DNA cytosine methyltransferase & Defence & QFQ04728.1 & 0 & BLASTp \\
\hline YdaU family protein & Unknown function & QFQ04729.1 & 0 & BLASTp \\
\hline $\begin{array}{c}\text { Replicative DNA helicase } \\
\text { (E.C.3.6.4.12), Bacteriophage; } \\
\text { Helicase-loader, Helicase, DNA } \\
\text { replication; HET: ADP; 4.1A } \\
\text { \{Escherichia coli O111:NM\}; Related } \\
\text { PDB entries: 6BBM_W 6BBM_V } \\
\text { 6BBM_X6BBM_Y }\end{array}$ & Replication & QFQ04730.1 & $1.20 \times 10^{-13}$ & HHpred \\
\hline Hypothetical protein & Unknown function & QFQ04731.1 & $6.00 \times 10^{-80}$ & BLASTp \\
\hline Hypothetical protein & Unknown function & QFQ04732.1 & $6.00 \times 10^{-109}$ & BLASTp \\
\hline Hypothetical protein & Unknown function & QFQ04733.1 & $2.00 \times 10^{-75}$ & BLASTp \\
\hline $\begin{array}{l}\text { DUF1064 domain-containing } \\
\text { protein }\end{array}$ & Unknown function & QFQ04734.1 & $4.00 \times 10^{-94}$ & BLASTp \\
\hline Antitermination protein & Replication & QFQ04735.1 & $4.00 \times 10^{-97}$ & BLASTp \\
\hline Hypothetical protein & Replication & QFQ04736.1 & $2.00 \times 10^{-32}$ & BLASTp \\
\hline Hypothetical protein & Unknown function & QFQ04737.1 & $6.00 \times 10^{-164}$ & BLASTp \\
\hline Hypothetical protein & Unknown function & QFQ04738.1 & $1.00 \times 10^{-60}$ & BLASTp \\
\hline $\begin{array}{l}\text { DNA mismatch endonuclease } \\
\text { (E.C.3.1.---)/DNA complex; } \\
\text { PROTEIN-DNA complex, } \\
\text { mismatch, intercalation, zinc; } 2.3 \mathrm{~A} \\
\{\text { Escherichia coli }\} \text { SCOP: c.52.1.15 }\end{array}$ & DNA repair & QFQ04739.1 & $9.50 \times 10^{-9}$ & HHpred \\
\hline
\end{tabular}


Table 4. Cont.

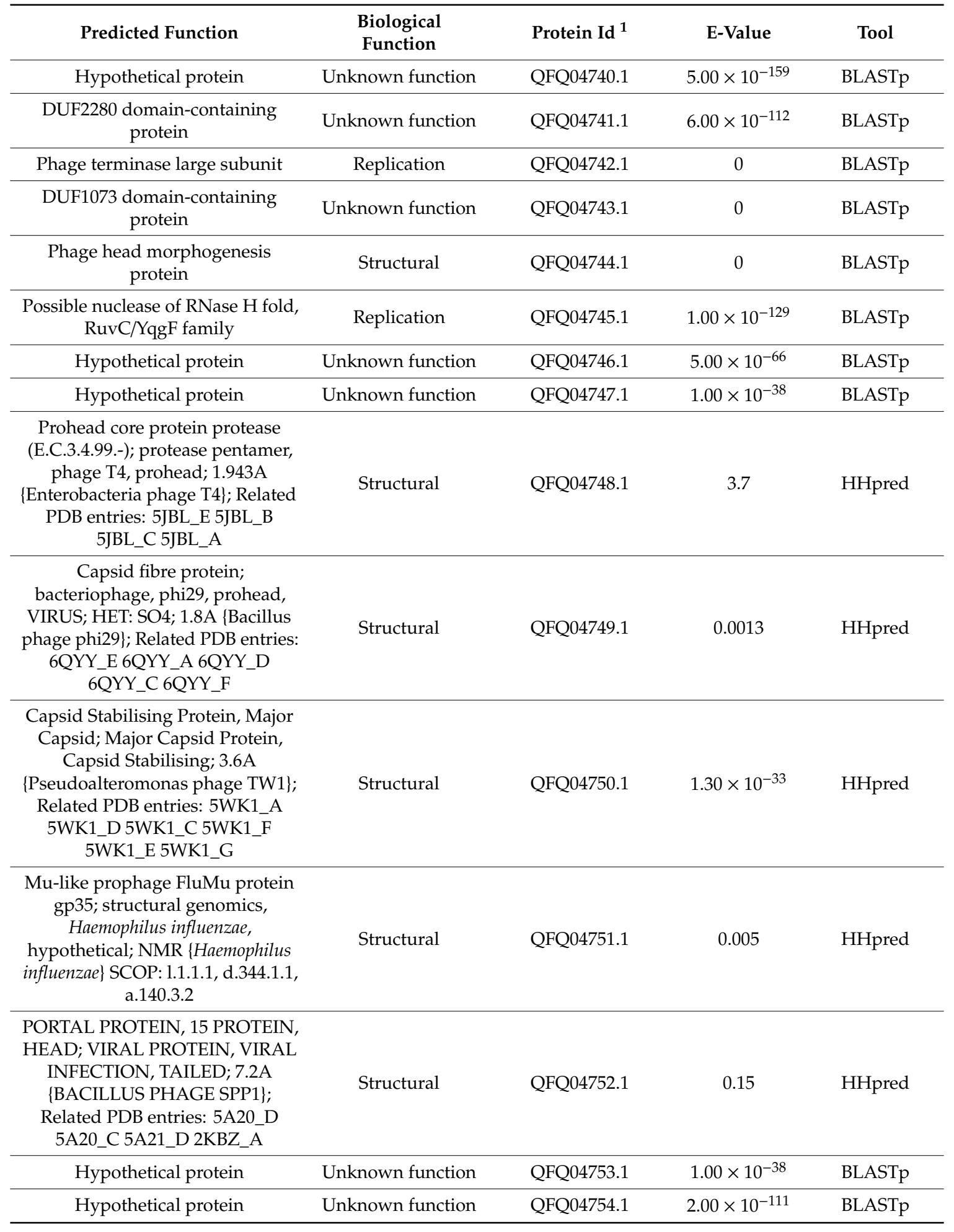


Table 4. Cont.

\begin{tabular}{|c|c|c|c|c|}
\hline Predicted Function & $\begin{array}{l}\text { Biological } \\
\text { Function }\end{array}$ & Protein Id $^{1}$ & E-Value & Tool \\
\hline $\begin{array}{c}\text { Minor tail protein U; Mixed } \\
\text { Alpha-Beta fold, VIRAL } \\
\text { PROTEIN; HET: MSE, SO4; 2.7A } \\
\text { \{Enterobacteria phage lambda\} } \\
\text { SCOP: d.323.1.1; Related PDB } \\
\text { entries: 3FZ2_L 3FZ2_K 3FZ2_F } \\
\text { 3FZB_E 3FZB_B 3FZB_A 3FZB_I } \\
\text { 3FZB_D 3FZB_J 3FZB_H 3FZB_C } \\
\text { 3FZ2_I 3FZB_F 3FZ2_A 3FZB_G } \\
\text { 3FZ2_D 3FZ2_J 3FZ2_H 3FZ2_E } \\
\text { 3FZ2_B 3FZ2_G 1Z1Z_A }\end{array}$ & Structural & QFQ04755.1 & $3.70 \times 10^{-17}$ & HHpred \\
\hline Hypothetical protein & Unknown function & QFQ04756.1 & 0 & BLASTp \\
\hline $\begin{array}{c}\text { Tail assembly chaperone; } \\
\text { Bacteriophage HK97, } \\
\text { morphogenesis, tail assembly; } \\
\text { HET: MSE; 2.3A \{Enterobacteria } \\
\text { phage HK97\}; Related PDB entries: } \\
\text { 2OB9_B }\end{array}$ & Structural & QFQ04757.1 & 6.5 & HHpred \\
\hline Hypothetical protein & Unknown function & QFQ04758.1 & $2.00 \times 10^{-48}$ & BLASTp \\
\hline $\begin{array}{l}\text { Zinc ribbon domain-containing } \\
\text { protein }\end{array}$ & Unknown function & QFQ04759.1 & $2.00 \times 10^{-117}$ & BLASTp \\
\hline Phage tail protein & Structural & QFQ04760.1 & 0 & BLASTp \\
\hline DUF2460 & Unknown function & QFQ04761.1 & $1.00 \times 10^{-161}$ & BLASTp \\
\hline $\begin{array}{l}\text { DUF2163 domain-containing } \\
\text { protein }\end{array}$ & Unknown function & QFQ04762.1 & 0 & BLASTp \\
\hline $\begin{array}{l}\text { SGNH/GDSL hydrolase family } \\
\text { protein (sialate } \\
\text { O-acetylesterase;cellulosome } \\
\text { enzyme) }\end{array}$ & Lysis & QFQ04763.1 & 0 & BLASTp \\
\hline $\begin{array}{c}\text { Putative Exo-alpha-sialidase; } \\
\text { Carbohydrate-Binding Module, } \\
\text { Bacterial Pathogen, Sialic; HET: } \\
\text { SIA; 2.2A \{CLOSTRIDIUM } \\
\text { PERFRINGENS\}; Related PDB } \\
\text { entries: 2V73_A }\end{array}$ & Lysis & QFQ04764.1 & 0.0032 & HHpred \\
\hline C40 family peptidase & Lysis & QFQ04765.1 & $2.00 \times 10^{-98}$ & BLASTp \\
\hline Putative phage tail protein & Structural & QFQ04766.1 & 0 & BLASTp \\
\hline Hypothetical protein & Unknown function & QFQ04767.1 & $4.00 \times 10^{-87}$ & BLASTp \\
\hline Glycosyl hydrolase 108 & Lysis & QFQ04768.1 & $2.00 \times 10^{-141}$ & BLASTp \\
\hline Anaerobic dehydrogenase & Lysis & QFQ04769.1 & $7.00 \times 10^{-73}$ & BLASTp \\
\hline Hypothetical protein & Unknown function & QFQ04770.1 & $4.00 \times 10^{-168}$ & BLASTp \\
\hline Y-family DNA polymerase & Defence & QFQ04771.1 & 0 & BLASTp \\
\hline $\begin{array}{l}\text { Trans-lesion error-prone DNA } \\
\text { polymerase V autoproteolytic } \\
\text { subunit }\end{array}$ & Defence & QFQ04772.1 & $6.00 \times 10^{-115}$ & BLASTp \\
\hline Site-specific integrase & Lysogeny & QFQ04773.1 & 0 & BLASTp \\
\hline
\end{tabular}


To determine whether our designation of the genomic region as a novel phage had functional relevance, we isolated vB_AbaS_LC1 after inducing its exit from the bacterial cell with mitomycin. Once vB_AbaS_LC1 was isolated and concentrated, we visualised it by TEM. TEM showed that the phage has a morphotype characteristic of a tailed phage from the Siphoviridae family, with a noncontractile tail of $250 \mu \mathrm{m}$ and an icosahedral capside of $60 \mu \mathrm{m}$ (Figure 4). Spot test results showed that vB_AbaS_LC1 has a narrow host range, as it was able to infect only two of the $19 \mathrm{~A}$. baumannii clinical strains assayed (Table S1). Each of the infected strains belonged to different STs, namely ST255 and ST265. Only one of the 19 strains assayed belonged to the same ST as ATCC 19606 (Ab22_GEIH2010; ST52), which was not susceptible to the virus.
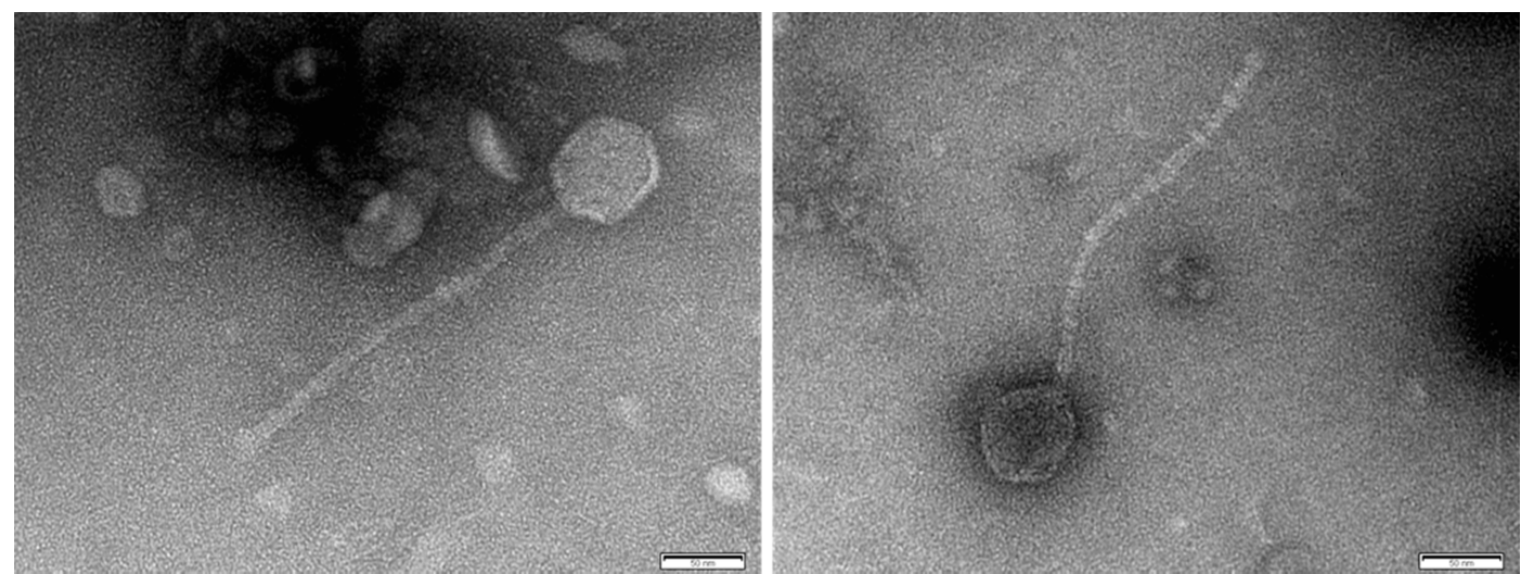

Figure 4. TEM image of the phage vB_AbaS_LC1. Samples of the phage in SM buffer were negatively stained with $1 \%$ aqueous uranyl acetate in grids and, then, were examined on a JEOL JEM-1011 electron microscope. Scale bar indicates $50 \mathrm{~nm}$ (nanometre).

\subsection{Biofilm Formation}

Biofilm formation is an important property that allows $A$. baumannii to survive on surfaces for a long period of time. To date, multiple genes have been shown to be involved in biofilm formation in A. baumannii, including ompA (encoding an outer membrane protein), the $c s u A B C D E$ operon (encoding a type I pili) and the bap and blp1 genes, which encode large biofilm-associated proteins and often vary in sizes [4,28]. Differences in the sizes of the proteins encoded by the bap and $b l p 1$ genes-differences that impact the presence/absence of domains present in the Bap and Blp1 proteins-have been used to explain some of the variations observed in the biofilm formations of A. baumannii strains. Knockouts that lack either of the bap and blp 1 genes produce significantly lower amounts of biofilm [4]. The complete genome of ATCC 19606 we report here encodes a 9831-bp blp1 gene (locus_id FQU82_03028) and an 18,543-bp bap gene (locus_id FQU82_03059). Both differ in size, mainly due to a different number of repeat units, compared to those encoded by AB0057 (GC1), ACICU (GC2) and ATCC 17978, which is another commonly used A. baumannii reference strain.

To complement the complete genome sequence of ATCC 19606 reported here, we quantified the biofilm formation of ATCC 19606 by crystal violet assay and by measuring biomass and thickness using fluorescence microscopy. A member of global clone 1 (AB0057), clone 2 (ACICU) and the widely used reference strain ATCC 17978 were also included in biofilm studies as controls. Using the standard crystal violet assay, and under the conditions examined here, visual inspection showed both ATCC 19606 and ATCC 17978 formed a little biomass at the culture-air interface compared to AB5007 and ACICU, which showed very strong staining around the well. These observations are reflected in the crystal violet measurements of OD600 0.78 and 0.36 versus 3.9 and 3.5, respectively. Using fluorescence microscopy, which captures cells fully submerged in the culture and directly attached to the surface, biofilm macro-colonies could be visualised in all strains after $24 \mathrm{~h}$. ATCC 19606 generated a biofilm biomass of approx. $1600 \mathrm{\mu m}^{3} / \mu^{2}{ }^{2}$, which was similar to that generated by ATCC 
17978 and higher than those generated by AB0057 and ACICU (Figure 5). Interestingly, after 48 h, ATCC 17978 showed the highest increase in biofilm biomass, while the biomass decreased in ATCC 19606 and other controls (Figure 5). Biofilm thickness was measured to be approx. $10 \mu \mathrm{m}$ for all strains after $24 \mathrm{~h}$, and all biofilms decreased by approx. 20\% in the 48-h experiment (Figure 5). Differences in biofilm formations observed in ATCC 19606 compared to other controls maybe explained by there being differences in the size of the proteins encoded by the blp 1 and bap genes between the strains tested.
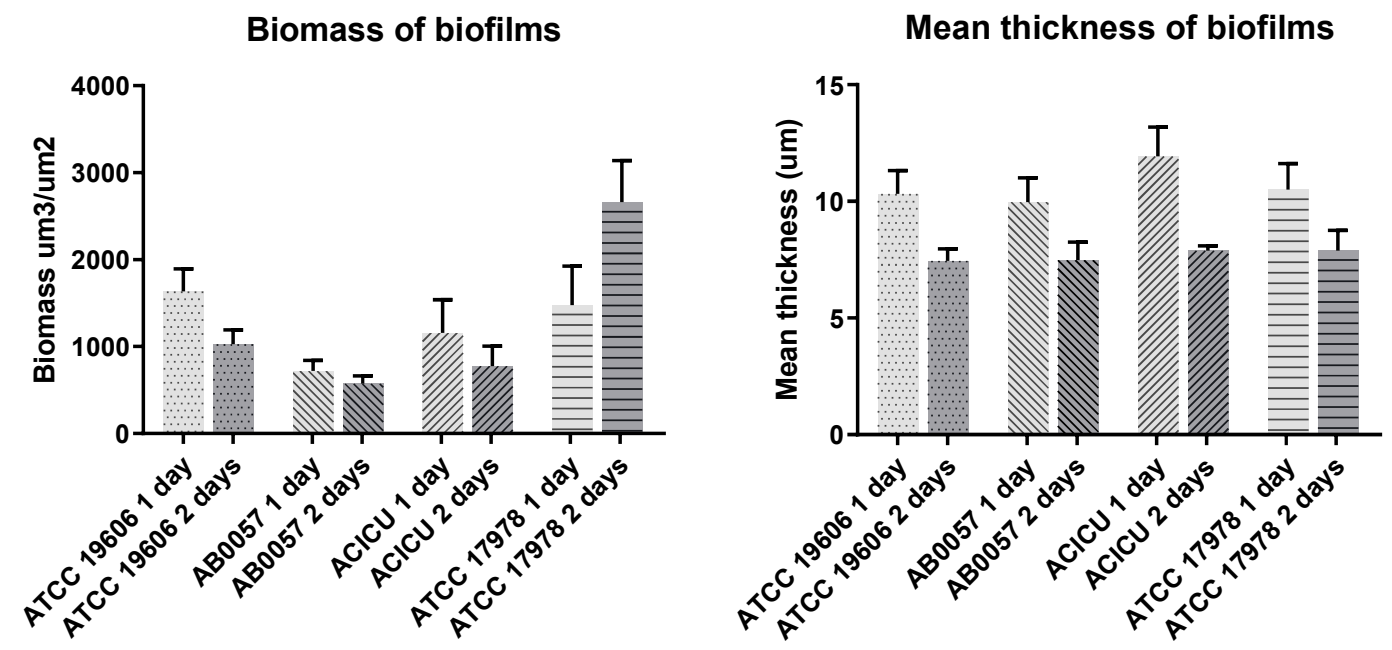

(a)
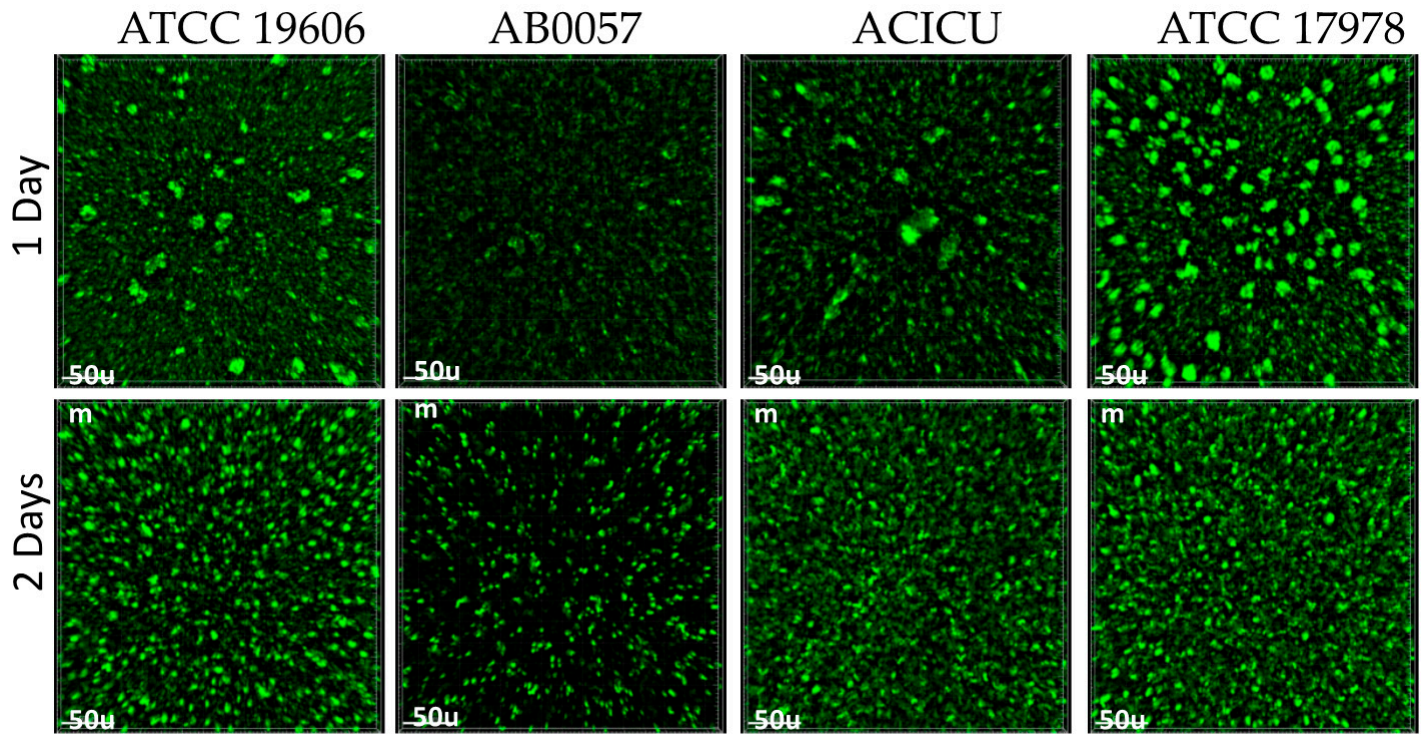

(b)

Figure 5. Analysis of the biofilms formed by ATCC 19606, ATCC 17078, AB5007 and ACICU using fluorescence microscopy. (a) shows the quantification of biofilm biomass and thickness of ATCC 19606 and controls after 24 and 48 h. (b) Fluorescence microscopy images of biofilms stained with Syto-9 and taken using the $20 \times$ lens after 24 and $48 \mathrm{~h}$ (top). 


\subsection{Surface Polysaccharide Loci Types}

Surface polysaccharides, including capsular polysaccharides (CPS, K or capsule), play a pathogenic role for A. baumannii. These loci are particularly important for the production of surface polysaccharides that function as virulence determinants for A. baumannii: the $\mathrm{K}$ locus that contains genes directing the synthesis of the surface polysaccharide capsular polysaccharide and the OC locus that contains genes involved in the synthesis of the outer core component of the lipooligosaccharide [29]. Previously, draft genomes of ATCC 19606 were used to predict the presence of the surface polysaccharide loci, including the KL3 capsule biosynthesis and OCL1 outer core [30]. Here, our assembly confirms the KL3 and OCL1 assignments.

\subsection{Comparative Analysis of ST52 Strains}

ATCC 19606 belongs to ST52. To examine the evolution of strains belonging to ST52, GenBank nonredundant and WGS (Whole Genome Shotgun) databases were explored and, then, phylogenetic analysis performed using the genomes found. In addition to ATCC 19606, 12 genomes were found as belonging to ST52 (Table 5). The additional genomes, all recovered after 2008, represent isolates from diverse geographical regions and diverse sources, including soil, clinical samples and hospital environments (Table 5). A phylogenetic analysis using the whole-genome alignment of ST52 strains showed, as expected, that they all cluster into a clade distinct from other major clonal groups, such as ST1 and ST2 (Figure 6).

However, a further analysis revealed a high degree of diversity within this tight phylogenetic group (Figure 6) -in particular, regarding their horizontally acquired resistance determinants. Within the ST52 clade, two distinct subclades were found. The subclade that we hereafter refer to as subclade 1 (SC1) contains a single Chinese strain (WE2714) in a deep branch and distant from the other strains. Subclade 2 (SC2) has two branches. In one branch, ATCC 19606, MSP4-16 and ab736 clustered tightly, indicating their close relationship, despite differences in their sources of isolation, country and isolation date (Table 5). The other branch of SC2 consists of a set of Japanese genomes, along with three additional genomes, one from Thailand and two from Pakistan. These three additional genomes cluster into a separate branch (Table 5 and Figure 6). Interestingly, the Japanese strains differed from each other by $<30$ single-nucleotide variants across their entire genomes, suggesting that they are likely to be outbreak strains given their isolation source and time (Table 5). 
Table 5. Properties of strains belonging to ST52 1 .

\begin{tabular}{|c|c|c|c|c|c|c|c|c|c|c|c|}
\hline Strain & Country & Date & Isolation Source & KL & OC & GI19606 & $\begin{array}{c}\text { Additional } \\
\text { Resistance Genes }\end{array}$ & $\operatorname{Tn} 6551$ & $\Phi^{3}$ & $\mathrm{p} 1 / \mathrm{p} 2^{4}$ & $\begin{array}{c}\text { GenBank } \\
\text { no. }\end{array}$ \\
\hline ATCC 19606 & US & $<1948$ & Urine & 3 & 1 & $\mathrm{Y}$ & - & + & + & $+/+$ & СР045110 \\
\hline MSP4-16 & India & 2010 & Mangrove soil & 3 & 1 & $\mathrm{Y}$ & - & + & + & $+/+$ & AODW01 \\
\hline ab736 & US & 2015 & Blood & 3 & 1 & $\mathrm{Y}$ & - & + & + & $-/+$ & СР015121 \\
\hline NBRC 110495 & Japan & 2008 & Human abscess & 3 & 1 & - & $a a d B^{5}$ & - & - & $-1+6$ & BBOR01 \\
\hline NBRC 110494 & Japan & 2008 & Burned skin & 3 & 1 & - & $a a d B^{5}$ & - & - & $-1+6$ & BBTE01 \\
\hline NBRC 110493 & Japan & 2008 & Burned skin & 3 & 1 & - & $a a d B^{5}$ & - & - & $-1+6$ & BBOQ01 \\
\hline GTC 03329 & Japan & 2008 & Human abscess & 3 & 1 & - & $a a d B^{5}$ & - & - & $-1+{ }^{6}$ & BBNJ01 \\
\hline GTC 03325 & Japan & 2008 & Burned skin & 3 & 1 & - & $a a d B^{5}$ & - & - & $-/+6$ & BBSP01 \\
\hline GTC 03324 & Japan & 2008 & Burned skin & 3 & 1 & - & $\begin{array}{c}\operatorname{aadB}^{5} \\
\text { tet }(B), \text { sul1, sul2, }\end{array}$ & - & - & $-1+{ }^{6}$ & BBNH01 \\
\hline AB_095 & Pakistan & 2016 & ICU washroom sink & 3 & 1 & - & $\begin{array}{c}\text { bla } a_{\mathrm{GES}-11,} \text { dfr } A 7, \\
\text { acc } 44, \text { oxa } 33, \text { aph } A 6\end{array}$ & - & - & $-1-$ & RHZR01 \\
\hline AB_165 & Pakistan & 2016 & $\begin{array}{l}\text { Alcohol foam } \\
\text { dispenser in ICU }\end{array}$ & 3 & 1 & - & 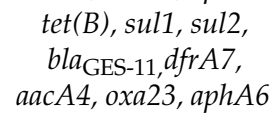 & - & - & $-/-$ & RHZA01 \\
\hline 4300STDY7045730 & Thailand & 2016 & na $^{7}$ & 57 & 1 & - & tet39 & - & - & $-1-$ & UFJF01 \\
\hline WE2714 & China & na & na & 32 & 6 & - & - & - & - & $-1-$ & QKVH01 \\
\hline
\end{tabular}

${ }^{1}$ All strains encode the OXA-694 variant of the intrinsic oxa-Ab gene. ${ }^{2}$ Additional antibiotic resistance genes (in addition to sul2, which is in the GIsul2 genomic island). ${ }^{3}$ vB_AbaS_LC1.

${ }^{4}$ p1, p1ATCC 19606 and p2, p2ATCC19606 (pMAC). ${ }^{5}$ aadB in plasmid pRAY*. ${ }^{6}$ An approx. 6-kb variant of p2ATCC 19606 (pMAC) present. ${ }^{7}$ Not available. 


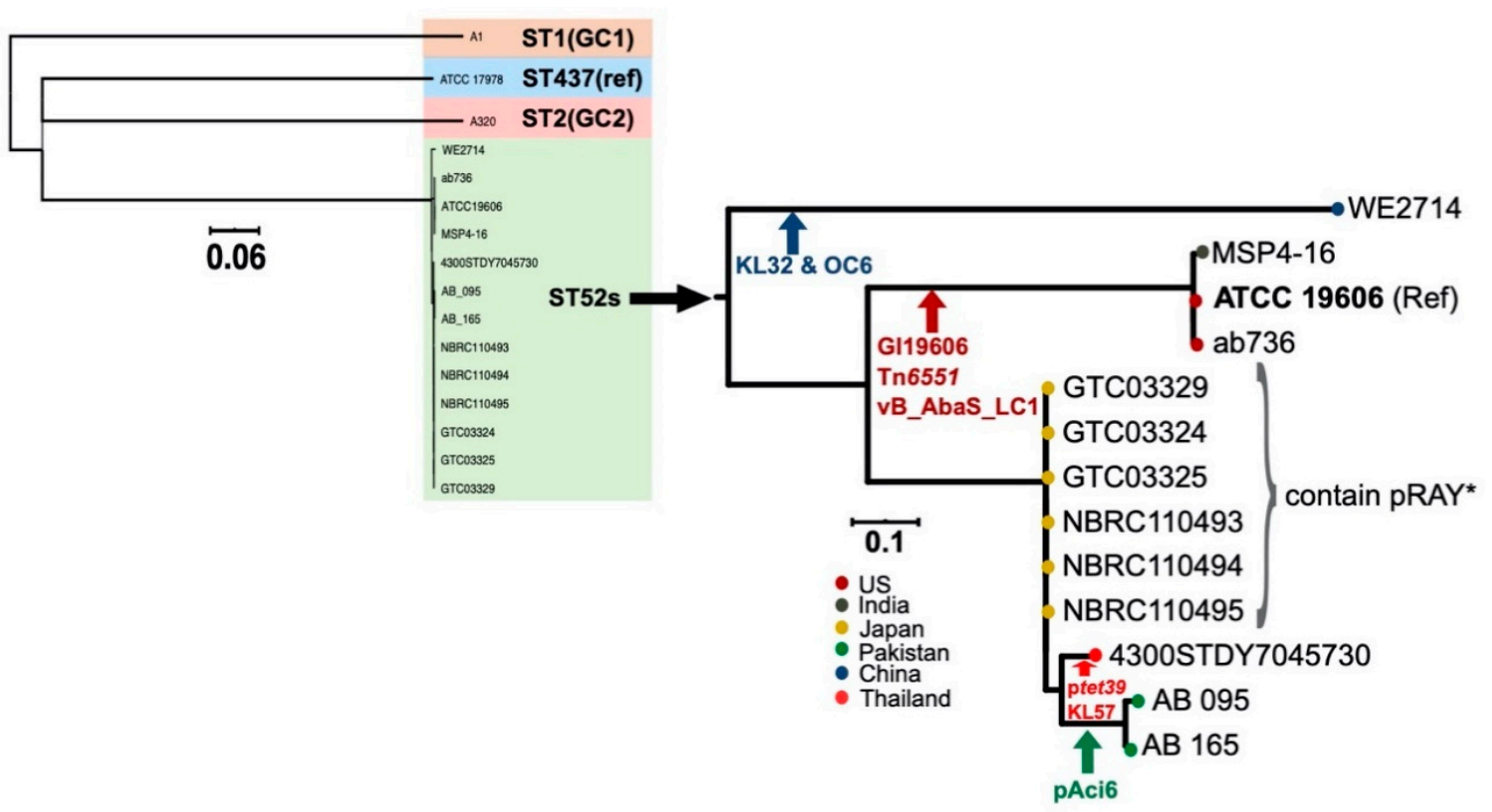

Figure 6. Phylogenetic relationship of ST52 strains to the references GC1, GC2 and ATCC 17978 (left) and within the sequence type 52 (right). Node colours indicate the country of isolation, and vertical arrows indicate branch-specific acquisition events. All Japanese strains indicated by a curly bracket contain $\mathrm{pRAY}^{*}$. Scale bars are shown.

We screened the ST52 strains for additional genetic features that could differentiate/group them. Interestingly, all strains in the ATCC 19606 branch, regardless of their country of origin and source of isolation, differ from other strains by the acquisition of the GI19606 genomic island, Tn6551 and the vB_AbaS_LC1 prophage (Figure 6), consistent with their phylogenetic placement in the same branch. All of the Japanese strains contain a copy of the small plasmid pRAY* (Figure 6 and Table 5). Variants of the small plasmid pRAY carry the aadB kanamycin, gentamicin and tobramycin resistance genes. So far, the $\mathrm{pRAY}^{*}$ variant has been mainly associated with members of global clone 1 [13,31]. The presence of pRAY* in all Japanese strains here (Figure 6 and Table 5) suggests a local acquisition, as the other strains in this subclade, which are from different countries, do not contain this plasmid. The Thai strain (4300STDY7045730) in SC2 contains a copy of the tetracycline-resistant tet39 gene. An analysis of this genome suggested that tet39 is located in a 9-kb plasmid, which appears to have a novel genetic structure. This was not pursued further. AB 095 and AB 165, both belonging to SC2, both recovered in Pakistan, contain several antibiotic resistance genes, including tet(B), sul1, sul2, bla $a_{\mathrm{GES}-11}$, dfrA7, aacA4, oxa23 and $a p h A 6$, predicting resistance to tetracycline, sulfamethoxazole, extended spectrum beta-lactam, trimethoprim and tobramycin, as well as carbapenems and amikacin, respectively. Interestingly, an analysis of $A B 095$ and $A B 165$ genomes indicated that all of the resistance genes are carried on a putative conjugative plasmid that encodes the RepAci6 replication initiation protein [32-34]. Plasmids that encode RepAci6 represent a group of conjugative plasmids that are mainly associated with the spread of the aphA6 amikacin and oxa23 carbapenem resistance genes [32-34]. They often carry aphA6 in a transposon called TnaphA6 and oxa23 either in Tn2006 or Tn2006 embedded in the AbaR4 resistance islands [32-34]. Our analysis predicted a copy of TnaphA6 and Tn2006 on a RepAci6 plasmid in AB 095 and AB 165. TnaphA6 was found precisely in the location previously identified in pAb-G7-2 (GenBank acc. no. KF669606) [33], and Tn2006 was in the location as in pK50 (GenBank acc. no. LT984690). The remainder resistance genes—tet(B), sul1, sul2, bla $a_{\mathrm{GES}-11}, d f r A 7$ and aacA4-were found in a MITE (Miniature Inverted-repeat Transposable Elements) region similar to that previously reported in p1AB5075 (GenBank acc. no. CP008707.1), which also encodes RepAci6 [35]. Together, the analysis of the antibiotic resistance genes in ST52 strains indicates that recent isolates have all become resistant to multiple antibiotics via different plasmids, further highlighting the significance of 
these mobile elements in the acquisition and spread of antibiotic resistance genes. The capsule and outer core surface polysaccharides (K and OC) loci are amongst the most variable genomic regions of A. baumannii genomes, even in closely related strains [30,36]. While the majority of ST52 strains encode the KL3 and OCL1 variants, WE2714, representing SC1, was found to contain KL32 and OCL6. A single strain in SC2 was also differed from other strains by a KL3àKL57 replacement (Figure 6).

\section{Discussion}

A. baumannii has emerged as an important opportunistic pathogen frequently associated with nosocomial infections - in particular, in intensive care units and in immunocompromised patients [1,2]. A. baumannii ATCC 19606 has been extensively used as a reference or model organism in studies involving the antibiotic resistance, virulence and pathogenesis of A. baumannii [37-41]. It is one of the earliest (isolated <1948) strains [5] available in current collections, making it an important strain for studies that involve antibiotic resistance. Access to high-quality complete genomes is especially important, as it provides insight into the malleability of plastic genomes; yet, until recently a complete genome for ATCC 19606 was not available. Thus far, there has also been no reported evidence of the evolutionary relationships and properties of members of ST52, a rare sequence type to which ATCC 19606 belongs. Such evidence can illuminate mechanisms for the development of antibiotic resistance, gene transfer between bacteria and increase of the resolution of outbreak tracing. Here, we report the characterisation of the first ATCC 19606 complete genome to be deposited in GenBank (October 2019) and present an analysis of its phenotypic and genomic features, as well as its evolutionary relationships to other ST52 strains.

In addition to the chromosome, our complete genome contains two cryptic plasmids, one of which (p2ATCC19606 or pMAC) was characterised in 2006 [27]. The ATCC 19606 genome completed by Zhu et al. [9] was missing the small 7-kbp cryptic plasmid (here, called p1ATCC19606), while the size and sequence of the second plasmid p2ATCC19606 (pMAC) was identical to our p2ATCC19606 sequence, as well as the original pMAC sequence (GenBank acc. no. AY541809.1). Although the ATCC 19606 genome completed by Tsubouchi et al. [10] contained p1ATCC19606 and p2ATCC19606, our analysis indicated both plasmids are likely to contain sequencing/assembling errors. In addition, given a large number of SNDs and short in/dels in this genome (GenBank acc. no. AP022836), and that p1ATCC19606 is missing from the genome completed by Zhu et al. [9], it shows that our assembly reported here is the most accurate genome for future studies of this strain.

In A. baumannii, antibiotic resistance genes are predominantly, although not exclusively, found within a large chromosomal genomic island. However, in more recent $A$. baumannii isolates, plasmids are significant in carrying and spreading antibiotic resistance genes [42-45]. We recently analysed a set of closely related carbapenem-resistant isolates that belong to GC1, lineage 2 and showed that they have become resistant to several antibiotics via six plasmids [46]. Similarly, here, we showed that more recent ST52 strains have also acquired genes conferring a resistance to several antibiotics via several plasmids, yet again highlighting the significance of these mobile elements in the acquisition and spread of antibiotic resistance determinants.

We characterised a novel cadmium/zinc/mercury transposon, Tn6551, in ATCC 19606 and showed it is related to Tn6018, a transposon commonly found in AbaR-type resistance islands in members of global clone 1 [3]. We previously showed that GC1 strains can gain ISAba125- and ISAba1-activated ampC genes [23] or an entire genomic island [22], along with a surrounding segment of the chromosome, by horizontal transfer via homologous recombination from an exogenous source. Here, we report another example where Tn6551, along with its flanking sequences (approx. $17 \mathrm{kbp}$ in total), were exchanged between ATCC 19606 and a ST638 strain, AB301.

To treat multi-resistant infections caused by A. baumannii strains, many studies have begun to characterise nonantibiotic approaches, including phage therapy, which has led to the characterisation of several phages with the potential to treat A. baumannii infections [47]. Here, we characterised 
a novel prophage, named vB_AbaS_LC1, which belongs to the Siphoviridae family, in ATCC 19606, with a potential to be used in phage therapy, although further work is required to confirm this.

Here, we present an accurate complete genome sequence of A. baumannii ATCC 19606, which can underpin future studies of A. baumannii. We also showed that ST52, to which ATCC 19606 belongs, is rare, with only 13 representatives in the GenBank nonredundant and WGS databases. Despite the popularity of ATCC 19606 as a model—driven initially by the early isolation and antibiotic susceptibility of ATCC 19606- in studies aimed at understanding the pathogenicity and virulence of A. baumannii, it might now be time to move away from ubiquitous reliance on ATCC 19606 to the selective use of this strain for specific purposes, given that strains belonging to ST52 are not a common cause of infections globally.

Supplementary Materials: The following are available online at http://www.mdpi.com/2076-2607/8/12/1851/s1: Table S1. Bacterial strains employed to determine the host range of the phage vB_AbaS_LC1.

Author Contributions: Conceptualisation, M.H.; methodology, M.H., L.N.T., L.B. and J.T.; software, M.H., L.N.T. and G.S.A.M.; validation, M.H.; formal analysis, M.H., M.T. and L.N.T.; investigation, M.H., L.B. and L.N.T.; resources, M.H., M.T. and G.S.A.M.; data curation, M.H. and L.N.T.; writing-original draft preparation, M.H.; writing - review and editing, M.H. and M.T.; visualisation, M.H.; supervision, M.H.; project administration, M.H. and funding acquisition, M.H and M.T. All authors have read and agreed to the published version of the manuscript.

Funding: This study and M.H. were supported by an Australian Research Council (ARC) DECRA fellowship (fellowship DE200100111). The prophage characterisation part was funded by grants PI16/01163 and PI19/00878 awarded to M. Tomás within the State Plan for R+D+I 2013-2016 (National Plan for Scientific Research, Technological Development and Innovation 2008-2011) and co-financed by the ISCIII-Deputy General Directorate for Evaluation and Promotion of Research-European Regional Development Fund "A way of Making Europe" and Instituto de Salud Carlos III FEDER.

Acknowledgments: We would like to thank Ruth Hall of the University of Sydney, Australia for supplying the strain ATCC 19606.

Conflicts of Interest: The authors declare no conflict of interest.

\section{References}

1. Peleg, A.Y.; Seifert, H.; Paterson, D.L. Acinetobacter baumannii: Emergence of a successful pathogen. Clin. Microbiol. Rev. 2008, 21, 538-582. [CrossRef] [PubMed]

2. Harding, C.M.; Hennon, S.W.; Feldman, M.F. Uncovering the mechanisms of Acinetobacter baumannii virulence. Nat. Rev. Microbiol. 2018, 16, 91-102. [CrossRef] [PubMed]

3. Hamidian, M.; Hall, R.M. The AbaR antibiotic resistance islands found in Acinetobacter baumannii global clone 1-Structure, origin and evolution. Drug Resist. Updates Rev. Comment. Antimicrob. Anticancer Chemother. 2018, 41, 26-39. [CrossRef] [PubMed]

4. De Gregorio, E.; Del Franco, M.; Martinucci, M.; Roscetto, E.; Zarrilli, R.; Di Nocera, P.P. Biofilm-associated proteins: News from Acinetobacter. BMC Genom. 2015, 16, 933. [CrossRef]

5. Hugh, R.; Reese, R. A comparison of 120 strains of Bacterium anitratum Schaub and Hauber with the type strain of this species. Int. J. Syst. Evol. Microbiol. 1968, 18, 207-229. [CrossRef]

6. Harris, G.; Lee, R.K.; Lam, C.K.; Kanzaki, G.; Patel, G.B.; Xu, H.H.; Chen, W. A mouse model of Acinetobacter baumannii-associated pneumonia using a clinically isolated hypervirulent strain. Antimicrob. Agents Chemother. 2013, 57, 3601-3613. [CrossRef]

7. Pachón-Ibáñez, M.E.; Docobo-Pérez, F.; López-Rojas, R.; Domínguez-Herrera, J.; Jiménez-Mejias, M.E.; García-Curiel, A.; Pichardo, C.; Jiménez, L.; Pachón, J. Efficacy of rifampin and its combinations with imipenem, sulbactam, and colistin in experimental models of infection caused by imipenem-resistant Acinetobacter baumannii. Antimicrob. Agents Chemother. 2010, 54, 1165-1172. [CrossRef]

8. Davenport, K.W.; Daligault, H.E.; Minogue, T.D.; Bruce, D.C.; Chain, P.S.; Coyne, S.R.; Jaissle, J.G.; Koroleva, G.I.; Ladner, J.T.; Li, P.E.; et al. Draft Genome Assembly of Acinetobacter baumannii ATCC 19606. Genome Announc. 2014, 2. [CrossRef] 
9. Zhu, Y.; Lu, J.; Zhao, J.; Zhang, X.; Yu, H.H.; Velkov, T.; Li, J. Complete genome sequence and genome-scale metabolic modelling of Acinetobacter baumannii type strain ATCC 19606. Int. J. Med. Microbiol. 2020, 310, 151412. [CrossRef]

10. Tsubouchi, T.; Suzuki, M.; Niki, M.; Oinuma, K.I.; Niki, M.; Kakeya,H.; Kaneko, Y.Complete Genome Sequence of Acinetobacter baumannii ATCC 19606(T), a Model Strain of Pathogenic Bacteria Causing Nosocomial Infection. Microbiol. Resour. Announc. 2020, 9. [CrossRef]

11. Wiegand, I.; Hilpert, K.; Hancock, R.E. Agar and broth dilution methods to determine the minimal inhibitory concentration (MIC) of antimicrobial substances. Nat. Protoc. 2008, 3, 163-175. [CrossRef] [PubMed]

12. CLSI. Performance Standards for Antimicrobial Susceptibility Testing; 22nd Informational Supplement; Clinical and Laboratory Standards Institute: Wayne, PA, USA, 2012.

13. Hamidian, M.; Hall, R.M. Genetic structure of four plasmids found in Acinetobacter baumannii isolate D36 belonging to lineage 2 of global clone 1. PLoS ONE 2018, 13, e0204357. [CrossRef] [PubMed]

14. O'Toole, G.A. Microtiter dish biofilm formation assay. J. Vis. Exp. 2011, 47. [CrossRef] [PubMed]

15. Gaio, D.; To, J.; Liu, M.; Monahan, L.; Anantanawat, K.; Darling, A.E. Hackflex: Low cost Illumina sequencing library construction for high sample counts. bioRxiv 2019. [CrossRef]

16. Wick, R.R.; Judd, L.M.; Gorrie, C.L.; Holt, K.E. Completing bacterial genome assemblies with multiplex MinION sequencing. Microb. Genom. 2017, 3, e000132. [CrossRef] [PubMed]

17. Wick, R.R.; Judd, L.M.; Gorrie, C.L.; Holt, K.E. Unicycler: Resolving bacterial genome assemblies from short and long sequencing reads. PLoS Comput. Biol. 2017, 13, e1005595. [CrossRef]

18. Seemann, T. Prokka: Rapid prokaryotic genome annotation. Bioinformatics 2014, 30, 2068-2069. [CrossRef]

19. Carver, T.J.; Rutherford, K.M.; Berriman, M.; Rajandream, M.A.; Barrell, B.G.; Parkhill, J. ACT: The Artemis Comparison Tool. Bioinformatics 2005, 21, 3422-3423. [CrossRef]

20. Kropinski, A.M.; Mazzocco, A.; Waddell, T.E.; Lingohr, E.; Johnson, R.P. Enumeration of bacteriophages by double agar overlay plaque assay. Methods Mol. Biol. 2009, 501, 69-76. [CrossRef]

21. Hamidian, M.; Hall, R.M. Acinetobacter baumannii ATCC 19606 Carries GIsul2 in a Genomic Island Located in the Chromosome. Antimicrob. Agents Chemother. 2017, 61. [CrossRef]

22. Hamidian, M.; Hawkey, J.; Wick, R.; Holt, K.E.; Hall, R.M. Evolution of a clade of Acinetobacter baumannii global clone 1, lineage 1 via acquisition of carbapenem- and aminoglycoside-resistance genes and dispersion of ISAba1. Microb. Genom. 2019, 5. [CrossRef] [PubMed]

23. Hamidian, M.; Hall, R.M. Resistance to third-generation cephalosporins in Acinetobacter baumannii due to horizontal transfer of a chromosomal segment containing ISAba1-ampC. J. Antimicrob. Chemother. 2014, 69, 2865-2866. [CrossRef]

24. Blackwell, G.A.; Hall, R.M. The tet39 Determinant and the msrE-mphE Genes in Acinetobacter Plasmids Are Each Part of Discrete Modules Flanked by Inversely Oriented pdif (XerC-XerD) Sites. Antimicrob. Agents Chemother. 2017, 61. [CrossRef] [PubMed]

25. D'Andrea, M.M.; Giani, T.; D’Arezzo, S.; Capone, A.; Petrosillo, N.; Visca, P.; Luzzaro, F.; Rossolini, G.M. Characterization of pABVA01, a plasmid encoding the OXA-24 carbapenemase from Italian isolates of Acinetobacter baumannii. Antimicrob. Agents Chemother. 2009, 53, 3528-3533. [CrossRef] [PubMed]

26. Merino, M.; Acosta, J.; Poza, M.; Sanz, F.; Beceiro, A.; Chaves, F.; Bou, G. OXA-24 carbapenemase gene flanked by XerC/XerD-like recombination sites in different plasmids from different Acinetobacter species isolated during a nosocomial outbreak. Antimicrob. Agents Chemother. 2010, 54, 2724-2727. [CrossRef] [PubMed]

27. Dorsey, C.W.; Tomaras, A.P.; Actis, L.A. Sequence and organization of pMAC, an Acinetobacter baumannii plasmid harboring genes involved in organic peroxide resistance. Plasmid 2006, 56, 112-123. [CrossRef] [PubMed]

28. Tomaras, A.P.; Dorsey, C.W.; Edelmann, R.E.; Actis, L.A. Attachment to and biofilm formation on abiotic surfaces by Acinetobacter baumannii: Involvement of a novel chaperone-usher pili assembly system. Microbiology 2003, 149, 3473-3484. [CrossRef] [PubMed]

29. Wyres, K.L.; Cahill, S.M.; Holt, K.E.; Hall, R.M.; Kenyon, J.J. Identification of Acinetobacter baumannii loci for capsular polysaccharide (KL) and lipooligosaccharide outer core (OCL) synthesis in genome assemblies using curated reference databases compatible with Kaptive. Microb. Genom. 2020, 6, e000339. [CrossRef]

30. Kenyon, J.J.; Hall, R.M. Variation in the complex carbohydrate biosynthesis loci of Acinetobacter baumannii genomes. PLoS ONE 2013, 8, e62160. [CrossRef] 
31. Hamidian, M.; Nigro, S.J.; Hall, R.M. Variants of the gentamicin and tobramycin resistance plasmid pRAY are widely distributed in Acinetobacter. J. Antimicrob. Chemother. 2012, 67, 2833-2836. [CrossRef]

32. Hamidian, M.; Hall, R.M. pACICU2 is a conjugative plasmid of Acinetobacter carrying the aminoglycoside resistance transposon TnaphA6. J. Antimicrob. Chemother. 2014, 69, 1146-1148. [CrossRef]

33. Hamidian, M.; Holt, K.E.; Pickard, D.; Dougan, G.; Hall, R.M. A GC1 Acinetobacter baumannii isolate carrying AbaR3 and the aminoglycoside resistance transposon TnaphA6 in a conjugative plasmid. J. Antimicrob. Chemother. 2014, 69, 955-958. [CrossRef]

34. Hamidian, M.; Kenyon, J.J.; Holt, K.E.; Pickard, D.; Hall, R.M. A conjugative plasmid carrying the carbapenem resistance gene blaOXA-23 in AbaR4 in an extensively resistant GC1 Acinetobacter baumannii isolate. J. Antimicrob. Chemother. 2014, 69, 2625-2628. [CrossRef]

35. Gallagher, L.A.; Ramage, E.; Weiss, E.J.; Radey, M.; Hayden, H.S.; Held, K.G.; Huse, H.K.; Zurawski, D.V.; Brittnacher, M.J.; Manoil, C. Resources for Genetic and Genomic Analysis of Emerging Pathogen Acinetobacter baumannii. J. Bacteriol. 2015, 197, 2027-2035. [CrossRef] [PubMed]

36. Holt, K.; Kenyon, J.J.; Hamidian, M.; Schultz, M.B.; Pickard, D.J.; Dougan, G.; Hall, R. Five decades of genome evolution in the globally distributed, extensively antibiotic-resistant Acinetobacter baumannii global clone 1. Microb. Genom. 2016, 2. [CrossRef] [PubMed]

37. Breisch, J.; Waclawska, I.; Averhoff, B. Identification and characterization of a carnitine transporter in Acinetobacter baumannii. MicrobiologyOpen 2019, 8, e00752. [CrossRef]

38. Moffatt, J.H.; Harper, M.; Harrison, P.; Hale, J.D.; Vinogradov, E.; Seemann, T.; Henry, R.; Crane, B.; St Michael, F.; Cox, A.D.; et al. Colistin resistance in Acinetobacter baumannii is mediated by complete loss of lipopolysaccharide production. Antimicrob. Agents Chemother. 2010, 54, 4971-4977. [CrossRef]

39. Sato, Y.; Unno, Y.; Kawakami, S.; Ubagai, T.; Ono, Y. Virulence characteristics of Acinetobacter baumannii clinical isolates vary with the expression levels of omps. J. Med. Microbiol 2017, 66, 203-212. [CrossRef]

40. Zeidler, S.; Müller, V. The role of compatible solutes in desiccation resistance of Acinetobacter baumannii. MicrobiologyOpen 2019, 8, e00740. [CrossRef]

41. Zimbler, D.L.; Arivett, B.A.; Beckett, A.C.; Menke, S.M.; Actis, L.A. Functional features of TonB energy transduction systems of Acinetobacter baumannii. Infect. Immun. 2013, 81, 3382-3394. [CrossRef] [PubMed]

42. Blackwell, G.A.; Hall, R.M. Mobilisation of a small Acinetobacter plasmid carrying an oriT transfer origin by conjugative RepAci6 plasmids. Plasmid 2019, 103, 36-44. [CrossRef] [PubMed]

43. Hamidian, M.; Ambrose, S.J.; Hall, R.M. A large conjugative Acinetobacter baumannii plasmid carrying the sul2 sulphonamide and strAB streptomycin resistance genes. Plasmid 2016, 87-88, 43-50. [CrossRef] [PubMed]

44. Leungtongkam, U.; Thummeepak, R.; Tasanapak, K.; Sitthisak, S. Acquisition and transfer of antibiotic resistance genes in association with conjugative plasmid or class 1 integrons of Acinetobacter baumannii. PLoS ONE 2018, 13, e0208468. [CrossRef] [PubMed]

45. Ma, F.; Shen, C.; Zheng, X.; Liu, Y.; Chen, H.; Zhong, L.; Liang, Y.; Liao, K.; Xia, Y.; Tian, G.B.; et al. Identification of a Novel Plasmid Carrying mor-4.3 in an Acinetobacter baumannii Strain in China. Antimicrob. Agents Chemother. 2019, 63. [CrossRef]

46. Douraghi, M.; Kenyon, J.J.; Aris, P.; Asadian, M.; Ghourchian, S.; Hamidian, M. Accumulation of Antibiotic Resistance Genes in Carbapenem-Resistant Acinetobacter baumannii Isolates Belonging to Lineage 2, Global Clone 1, from Outbreaks in 2012-2013 at a Tehran Burns Hospital. mSphere 2020, 5. [CrossRef]

47. García-Quintanilla, M.; Pulido, M.R.; López-Rojas, R.; Pachón, J.; McConnell, M.J. Emerging therapies for multidrug resistant Acinetobacter baumannii. Trends Microbiol. 2013, 21, 157-163. [CrossRef]

Publisher's Note: MDPI stays neutral with regard to jurisdictional claims in published maps and institutional affiliations.

(C) 2020 by the authors. Licensee MDPI, Basel, Switzerland. This article is an open access article distributed under the terms and conditions of the Creative Commons Attribution (CC BY) license (http://creativecommons.org/licenses/by/4.0/). 